\title{
Optimal Prevention and Treatment Control on SVEIR Type Model Spread of COVID-19
}

\author{
Jonner Nainggolan \\ Department of Mathematics, Cenderawasih University Jayapura Indonesia \\ Email: jonner2766@gmail.com
}

\begin{abstract}
COVID-19 pandemic has disrupted the world's health and economy and has resulted in many deaths since the first case occurred in China at the end of 2019. Moreover, The COVID-19 disease spread throughout the world, including Indonesia on March 2, 2020. Coronavirus quickly spreads through droplets of phlegm through the throat to the lungs. Researchers in the medical field and the exact science are jointly examined transmission, prevention, and optimal control of COVID-19 disease. Due to the prevention of COVID-19, a vaccine has been found in early 2021, which at the time, the vaccination process was carried out worldwide against COVID-19. This paper examines the spread model of SVEIR-type COVID-19 by considering the vaccination subpopulation. In this study, control of prevention efforts $\left(u_{1}^{*}\right.$ and $\left.u_{2}^{*}\right)$ and healing efforts $\left(u_{3}^{*}\right)$ are given and being analyzed with the fourth-order Runge-Kutta approach. Based on numerical simulations, it can be seen that using the controls $u_{1}^{*}, u_{2}^{*}$ and $u_{3}^{*}$ can decrease the amount of infected people in the subpopulation compared to those without control. The $u_{3}^{*}$ control can increase the number of recovered individual subpopulations.
\end{abstract}

Keywords: COVID-19; SVEIR model; optimal control; treatment; vaccination.

\section{INTRODUCTION}

Coronavirus is a virus that attacks the respiratory system. Corona virus interferes with mild respiratory and lung infections and can result in death [1]. Corona virus is rapidly spreading to almost all countries in the world, and Indonesia on March 2, 2020. To prevent the spread of COVID-19, the government recommends frequent hand washing with soap/hand sanitizer and practicing cough etiquette. Corona virus spreads by sprinkling phlegm from the throat of an infected person, especially in closed air circulation areas. Be aware of COVID-19, improve your health with healthy lifestyle, includes: balanced nutrition consumption, exercises, adequate rest, and frequent handwashing with soap.

Exact science and medicine experts are working together to prevent the spread of COVID-19. Consequently, Mathematical researchers are also take part in assessing transmission, streamlining, and optimizing control of the disease. The optimal control strategy for the control of pandemic avian influenza along with the quarantine subpopulation [2]. Studying the model of the spread of the Corona Virus type SEIR in Wuhan, by controlling people's travel history to and from the city of Wuhan [3]. Then, compared to the pattern of the spread of COVID-19 in Wuhan, China, and Internationally in January and February 2020. Examine the mathematical modeling of the epidemic of COVID-19 cases in Nigeria from 29 
March to 12 June 2020 with the effect of public awareness programs in implementing health protocols according to government recommendations [4]. The model for the spread of COVID19 by paying attention to symptomatically and asymptomatically infectious compartments [5]. Studied the spread of the COVID-19 outbreak by applying mathematical growth functions and analyzing cases caused by the disease [6]. Examined a mathematical model on the SIR type of COVID-19 to predict its spread by considering the social distancing factors [7]. Formulated a deterministic model on the spread of COVID-19 by estimating the model parameters according to the pandemic data that occurred in India, then conducting a sensitivity analysis to identify the model parameters [8]. Analyzed the COVID-19 modeling based on morbidity data in Anhui, China by taking into account increased morbidity and mitigation measures [9]. Studied and analyzed Corona Virus disease spread system and the SEIR type Cov-2 SAR with the effectiveness of government intervention [10]. Analyzed model and predicted the spread of COVID-19, then examined the exposed subpopulation, with measurement to prevent and control the epidemic [11]. Examined the growth of the logistic model of the COVID-19 spread in China and compared with data globally, determined the parameter values with a least-squares approach [12].

Studied a mathematical model for the COVID-19 pandemic, type SIR, with asymptomatic individual effects considered with the finite antibody duration and Health Policy [13]. Studied the global dynamics of the SEIR type COVID-19 under convex incidence rate, through a mathematical model [14]. Other studies have been carried out to find the exponential growth rate of the epidemic and determine the basic reproduction number [15]. Reviewed A nonlinear ordinary differential equation model for the spread of COVID-19, the model studied predicts the total number of COVID-19 cases in Austria, France, and Poland [16]. The model studied is based on the model studied by Fang et al. (2020), figuring out the vaccinated subpopulation and providing optimal control of $u_{1}, u_{2}$, and $u_{3}$. The objectives of this study includes: (1) To examine and analyze the SVEIR-type of COVID-19 spread model, (2) determine the co-state function of the SVEIR-type of COVID-19 spread model with control $u_{1}^{*}, u_{2}^{*}$ and $u_{3}^{*}$. (3) Determine numerical solution of the optimal control for prevention and treatment of the SVEIR-type of COVID-19 spread model. Optimal control of prevention and treatment in the model of the spread of COVID19 is important to study, since the COVID-19 disease is still spreading and has not been completely the treatment until now. This study will examine the SVEIR type of COVID-19 spread model by considering vaccination subpopulations and provide preventive control measures $\left(u_{1}^{*}\right.$ and $\left.u_{2}^{*}\right)$, healing efforts $\left(u_{3}^{*}\right)$ and analyzed simulation by numerical approach using Runge-Kutta fourth order.

\section{METHOD}

The model used in this study comes from the development of the Tian (2020) which contemplated the recruitment of suspected subpopulations denoted by S, namely individuals who have a history of travel to infected areas. Vaccination subpopulation, namely susceptible individuals who are vaccinated to increase individual immunity to the COVID-19 virus so as not to be infected with COVID-19 disease. The exposed subpopulation, namely individuals who are positive for COVID-19 from the results of the swab, but not severe, denoted $\mathrm{E}$. Infected subpopulations denoted by I, i.e. individuals who are positive for COVID-19 from the swab results and experience severe illness due to COVID-19. The recovered subpopulation is denoted by $\mathrm{R}$, due to treatment in hospitals provided by the Government or due to self-isolation at home by eating or taking vitamins to increase immunity so that they can recover from COVID-19. The assumptions of the 
model studied are as follows: (i) Individuals who travel to areas infected with COVID-19 or have been in contact with individuals infected with COVID-19 enter the susceptible subpopulation. (ii) Does not consider the natural mortality of each subpopulation. (iii) Most of the susceptible individuals who want to be vaccinated yet entering the vaccination subpopulation.

(iv) Individuals who were successfully vaccinated entered the recovered subpopulation, and those who failed to enter the exposed subpopulation. (v) If the maximum number of individuals in the subpopulation is exposed or the virus is multiplying in the lungs, then the individual will enter the infected subpopulation. (vi) Pay attention to deaths due to COVID-19. (vi) Individuals may experience recovery due to treatment or by self-isolation.

The models of the spread of COVID-19 studied are as follows:

$$
\begin{aligned}
& \frac{d S}{d t}=\Lambda-\frac{\beta S I}{N}-\theta S \\
& \frac{d V}{d t}=\theta S-(\sigma+r) V \\
& \frac{d E}{d t}=\frac{\beta S I}{N}+\sigma V-\gamma E \\
& \frac{d I}{d t}=\gamma E-(d+\delta+\tau) I \\
& \frac{d R}{d t}=r V+(\delta+\tau) I
\end{aligned}
$$

\begin{tabular}{|c|c|c|c|}
\hline Parameter & Description & Value & Reference \\
\hline$\Lambda=\eta N$ & Recruitment rate entering the $S$ subpopulation & $0,047 /$ day & $\begin{array}{l}\text { COVID-19 } \\
\text { go.id data }\end{array}$ \\
\hline$\beta$ & Transmission rate from $S$ to $E$ & $0,154 /$ day & Assumed \\
\hline$\theta$ & Vaccination rates from subpopulation $\mathrm{S}$ to $V$ & $0,04 /$ day & Assumed \\
\hline$\sigma$ & $\begin{array}{l}\text { The transfer rate from subpopulation } V \text { to } E \text {, due to } \\
\text { vaccine failure }\end{array}$ & $0,005 /$ day & Assumed \\
\hline$r$ & Vaccination success rate & $0,05 /$ day & Assumed \\
\hline$\gamma$ & Transmission rate from subpopulation $E$ to $I$ & $0,036 /$ day & Assumed \\
\hline$d$ & Death rate due to COVID-19 & $0,002 /$ day & $\begin{array}{l}\text { COVID-19 } \\
\text { go.id data }\end{array}$ \\
\hline$\delta$ & Healing rate & 0,036/day & $\begin{array}{l}\text { COVID-19 } \\
\text { go.id data }\end{array}$ \\
\hline$\tau$ & Speed of healing with COVID-19 self-isolation & $0,04 /$ day & Assumed \\
\hline
\end{tabular}

Where $N=S+V+E+I+R$. Description of the parameters as Table 1 follows:

Table 1. Parameter description and estimate value

\section{Equilibrium Point}

Non-endemic fixed point, to analyze equation (1)-(5) it is enough to use equation (1)-(4) because equation (5) is redundant to equation (1)-(4). Based on equation (1)-(4), the non-endemic fixed point is obtained, namely:

$$
E_{0}=\left(\frac{\Lambda}{\theta}, \frac{\Lambda}{r+\sigma}, 0,0\right) \text {, }
$$

and the endemic point of system (1)-(4) is

$$
E_{1}=\left(\frac{\Lambda N}{\beta I+\theta N}, \frac{\theta \Lambda N}{(r+\sigma)(\beta I+\theta N)}, \frac{\beta \Lambda N}{\gamma(\beta I+\theta N)}+\frac{\sigma \theta \Lambda N}{\gamma(r+\sigma)(\beta I+\theta N)}, I^{* *}\right)
$$

with $I^{* *}=-\frac{1}{2} \frac{c \theta N T \pm \sqrt{(c \theta N T)^{2}+4 a \beta c^{2} T+4 b \beta c T}}{c \beta T}, a=\beta \Lambda N, b=\sigma \theta \Lambda N, T=d+\delta+\tau$, $c=r+\sigma$. 


\section{The Reproduction Number}

The reproduction number is a parameter that expresses the expectation of secondary infective individuals due to contracting primary infective individuals in the susceptible population. The standard parameters that need to be known to determine whether the disease is spreading or not are the basic reproduction number $\left(\boldsymbol{R}_{0}\right)$, if $\boldsymbol{R}_{\boldsymbol{v}}>1$ then the number of infected individuals increases, if $\boldsymbol{R}_{0}<1$ then the number of infected individuals does not increase or decrease. The basic reproduction number is calculated by using the next-generation method, the basic reproduction number of equations (1)(5) are as follows:

$$
\boldsymbol{R}_{0}=\rho\left(G U^{-1}\right)
$$

with $\rho$ is radius of the matrix built by $G U^{-1}$. The rate of new infections denoted with $G$, and $U$ is individuals out. The Jacobian matrix of $G(x)$ and $U(x)$, and denote $G=\left[\partial G_{i} / \partial \mathrm{x}_{\mathrm{j}}\right]$ and $U$ $=\left[\partial \mathrm{U}_{i} / \partial \mathrm{x}_{\mathrm{j}}\right],(i, j=1,2,3,4,5)$. Parameter the reproduction number, $\boldsymbol{R}_{\boldsymbol{O}}$ as

$$
\boldsymbol{R}_{\boldsymbol{O}}=\frac{r \beta \Lambda}{\theta N(d+\delta+\tau)(r+\sigma)} \text {. }
$$

\section{RESULT AND DISCUSSION}

The handling of COVID-19 carried out by the stakeholders are: Control $u_{1}(t)$ for prevention with government appeals in government agencies, schools, and the general public by implementing physical distancing, namely maintaining a minimum distance of 1 meter from other people. Then, implementing a mask when doing activities in public places or crowds. To continue, washing our hands regularly with soap and water or a hand sanitizer that contains at least $60 \%$ alcohol, especially after coming back from outdoor activities or in public places. The $u_{2}(t)$ control is vaccination counseling because many individuals are afraid to be vaccinated. This $u 2(t)$ control provides education to the public, how to prepare for vaccination, what to do after being vaccinated, and what are the effects after being vaccinated. Because there is a lot of hoax information circulating, to scare people not to be vaccinated. $U_{3}(t)$ control is an effort to accelerate healing from COVID-19 by individuals who are self-isolating in their own homes or places provided by the central government or local governments. The healing efforts given are: taking vitamins $C, D, B$, zinc, selenium, curcumin, Echinacea.

Based on equations (1)-(5) after being given the control $u_{1}(t), u_{2}(t)$ and $u_{3}(t)$ obtained a system of differential equations with optimal control as follows:

$$
\begin{aligned}
& \frac{d S}{d t}=\Lambda-\frac{\beta\left(1-u_{1}(t)\right) S I}{N}-\theta\left(1+u_{2}(t)\right) S \\
& \frac{d V}{d t}=\theta\left(1+u_{2}(t)\right) S-(\sigma+r) V \\
& \frac{d E}{d t}=\frac{\beta\left(1-u_{1}(t)\right) S I}{N}+\sigma V-\gamma E \\
& \frac{d I}{d t}=\gamma E-\left(d+\delta+\tau\left(1+u_{3}(t)\right)\right) I \\
& \frac{d R}{d t}=r V+\left(\delta+\tau\left(1+u_{3}(t)\right)\right) I
\end{aligned}
$$

The functional objective of optimal control studied in this paper are:

$$
J\left(u_{1}, u_{2}, u_{3}\right)=\int_{0}^{t_{f}}\left(A E(t)+B I(t)+C_{1} u_{1}^{2}(t)+C_{2} u_{2}^{2}(t)+C_{3} u_{3}^{2}(t)\right) d t
$$

where the coefficients $A, B$ is the balance weights of the individual compartments exposed and actively infected with COVID-19, respectively. While the coefficient $C_{1}$ is a parameter weight that corresponds to the control $u_{1}(t), C_{2}$ is a parameter weight that corresponds to the control $u_{2}(t)$, and $C_{3}$ is a parameter weight corresponding to the control $u_{3}(t)$, and $t_{f}$ is 
the end time of the period. Let $u_{1}^{*}(t), u_{2}^{*}(t)$, and $u_{3}^{*}(t)$, be the optimal control of the system (7)-(11) and (12), such that it satisfies

$$
J\left(u_{1}^{*}, u_{2}^{*}, u_{3}^{*}\right)=\min _{\kappa} J\left(u_{1}, u_{2}, u_{3}\right) \text {, }
$$

where the control set

$$
U=\left\{\left(u_{1}, u_{2}, u_{3}\right) \mid u_{i}:\left[0, t_{f}\right] \rightarrow[0,1] \text {, Lebesgue Measurable, } i=1,2,3\right\} .
$$

The objective function (12), the optimal control $u_{1}^{*}, u_{2}^{*}, u_{3}^{*}$, obtained with provided that (13) with restriction system (7)-(11) by using matlab tools, the solution will be obtained [17]. By model (7)-(11), and minimum functional adjoint (12) obtained Hamiltonian function $H$, that is

$$
H=A E+B I+C_{1} u_{1}^{2}+C_{2} u_{2}^{2}+C_{3} u_{3}^{2}+\lambda_{1} \frac{d S}{d t}+\lambda_{2} \frac{d V}{d t}+\lambda_{3} \frac{d E}{d t}+\lambda_{4} \frac{d I}{d t}+\lambda_{5} \frac{d R}{d t}
$$

\section{Theorem 1}

There exists a optimal control $u_{1}^{*}(t), u_{2}^{*}(t)$ and $u_{3}^{*}(t)$ and associated solution $S^{*}(t), V^{*}(t), E^{*}(t), I^{*}(t), R^{*}(t)$ from models (7)-(11) and (14). Then there exist costate functions $\lambda_{i}, i=1,2,3,4,5$ satisfying

$$
\begin{aligned}
& \frac{d \lambda_{1}}{d t}=\left(\lambda_{1}-\lambda_{3}\right) \frac{\beta\left(1-u_{1}\right) I}{N}+\left(\lambda_{1}-\lambda_{2}\right)\left(1+u_{2}\right) \theta \\
& \frac{d \lambda_{2}}{d t}=\left(\lambda_{2}-\lambda_{3}\right) \sigma+\left(\lambda_{2}-\lambda_{5}\right) r \\
& \frac{d \lambda_{3}}{d t}=-A+\left(\lambda_{3}-\lambda_{4}\right) \gamma \\
& \frac{d \lambda_{4}}{d t}=-B+\left(\lambda_{1}-\lambda_{3}\right) \frac{\beta\left(1-u_{1}\right) S}{N}+\left(\lambda_{4}-\lambda_{5}\right)(\delta+\tau)+\lambda_{4} d \\
& \frac{d \lambda_{5}}{d t}=0,
\end{aligned}
$$

The transversality conditions are given by $\lambda_{i}\left(t_{f}\right)=0, i=1,2,3,4,5$. Finally, from the optimality condition, we obtain the following optimal controls:

Proof:

$$
\begin{aligned}
& u_{1}^{*}=\min \left\{\max \left\{0, \frac{\left(\lambda_{3}-\lambda_{1}\right) \beta S I}{2 C_{1} N}\right\}, 1\right\} \\
& u_{2}^{*}=\min \left\{\max \left\{0, \frac{\left(\lambda_{1}-\lambda_{2}\right) \theta S}{2 C_{2}}\right\}, 1\right\} . \\
& u_{3}^{*}=\min \left\{\max \left\{0, \frac{\left(\lambda_{4}-\lambda_{5}\right) \tau I}{2 C_{3}}\right\}, 1\right\} .
\end{aligned}
$$

We use Pontrygain's Maximum Principle [17] on our model system (14), and the Hamiltonian is given by,

$$
\begin{aligned}
H=A I & +B_{1} u_{1}^{2}+B_{2} u_{2}^{2}+\lambda_{1}\left(\Lambda-\frac{\beta\left(1-u_{1}(t)\right) S I}{N}-\theta\left(1+u_{2}(t)\right) S\right) \\
& +\lambda_{2}\left(\theta\left(1+u_{2}(t)\right) S-(\sigma+r) V\right)+\lambda_{3}\left(\frac{\beta\left(1-u_{1}(t)\right) S I}{N}+\sigma V-\gamma E\right)+ \\
& +\lambda_{4}\left(\gamma E-\left(d+\delta+\tau\left(1+u_{3}(t)\right)\right) I\right)+\lambda_{5}\left(r V+\left(\delta+\tau\left(1+u_{3}(t)\right)\right) I\right) \\
\frac{d \lambda_{1}}{d t}= & -\frac{\partial H}{\partial S} \\
= & \left(\lambda_{1}-\lambda_{3}\right) \frac{\beta\left(1-u_{1}\right) I}{N}+\left(\lambda_{1}-\lambda_{2}\right)\left(1+u_{2}\right) \theta \\
\frac{d \lambda_{2}}{d t=} & -\frac{\partial H}{\partial V} \\
= & \left(\lambda_{2}-\lambda_{3}\right) \sigma+\left(\lambda_{2}-\lambda_{5}\right) r \\
\frac{d \lambda_{3}}{d t}= & -\frac{\partial H}{\partial E} \\
= & -A+\left(\lambda_{3}-\lambda_{4}\right) \gamma \\
\frac{d \lambda_{4}}{d t}= & -\frac{\partial H}{\partial I}
\end{aligned}
$$




$$
\begin{aligned}
& =-B+\left(\lambda_{1}-\lambda_{3}\right) \frac{\beta\left(1-u_{1}\right) S}{N}+\left(\lambda_{4}-\lambda_{5}\right)(\delta+\tau)+\lambda_{4} d \\
\frac{d \lambda_{5}}{d t} & =-\frac{\partial H}{\partial R} \\
& =0 .
\end{aligned}
$$

The optimality equations (14) and must satisfy transversality conditions $\lambda\left(t_{f}\right)=0$ for values $i=1,2,3,4,5$. There exist unique optimal controls $u_{1}^{*}(t)$ and $u_{2}^{*}(t)$ which minimize $J$ over $U$ : The optimality necessary conditions that $\frac{\partial H}{\partial u_{1}}=0, \frac{\partial H}{\partial u_{2}}=0$ and $\frac{\partial H}{\partial u_{3}}=0$, then, by the bounds on the controls, it is easy to obtain and in the form $u_{1}^{*}(t)=\frac{\left(\lambda_{3}-\lambda_{1}\right) \beta S I}{2 C_{1} N}$ , $u_{2}^{*}(t)=\frac{\left(\lambda_{1}-\lambda_{2}\right) \theta S}{2 C_{2}}$, and $u_{3}^{*}(t)=\frac{\left(\lambda_{4}-\lambda_{5}\right) \tau I}{2 C_{3}}$. The optimal prevention control of disease, the reproduction numbers declared to be as follows:

$$
R_{0 p}^{*}=\frac{r \beta\left(1-u_{1}\right) \Lambda}{\theta N\left(1+u_{2}\right)(d+\delta+\tau)(r+\sigma)} .
$$

The optimal healing control of disease, the reproduction numbers declared to be as follows:

$$
R_{0 h}^{*}=\frac{r \beta \Lambda}{\theta N\left(d+\delta+\tau\left(1+u_{3}\right)\right)(r+\sigma)} .
$$

\section{Numerical Method}

To solve the optimal control on the studied system of differential equations, it is solved by using a numerical method approach. The numerical solution used is the Pontryagin Maximum Principle. Completion of the optimal control system using the fourth-order Runge-Kutta procedure iterative method. The solution of the model (7)-(11) by guessing the initial and forward time from left to right with the same time co-state is solved from left to right by a forward Runge-Kutta fourth-order procedure in time with conditions of transversality [1]. Suppose the initial number of subpopulations $S_{0}=61478$ , $V_{0}=40000$ (assumed), $E_{0}=20000$ (assumed), $I_{0}=26940, R_{0}=7637$.

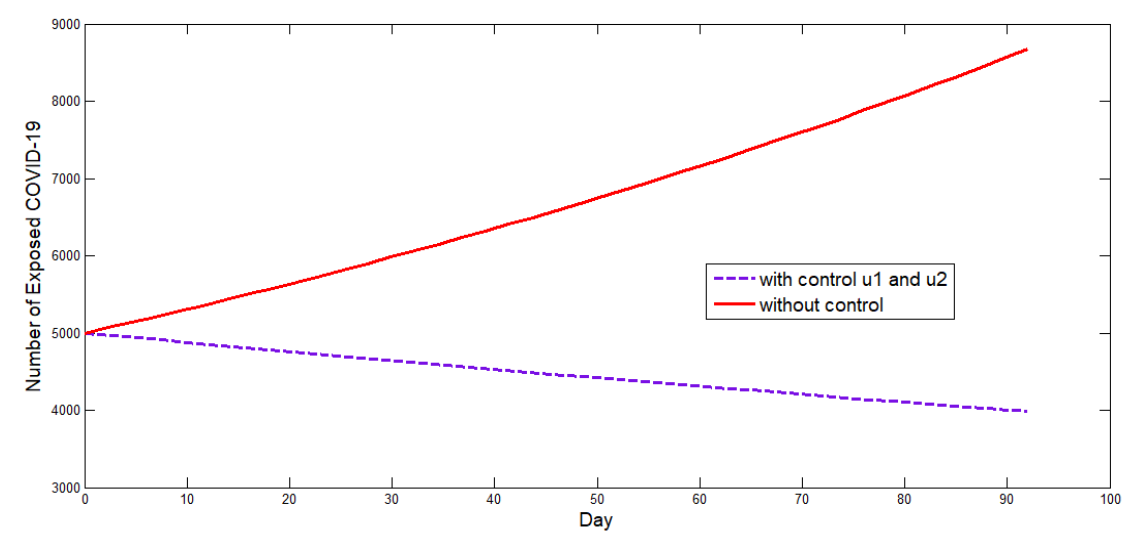

Figure 1. The dynamics of $E$ with $u_{1}^{*}$ and $u_{2}^{*}$ controls

Figure 1, the optimal control on COVID-19 prevention $\left(u_{1}^{*}\right)$ and on Efforts to increase the effectiveness of vaccination $\left(u_{2}^{*}\right)$ are used equation (12). The results in Figure 1 show that a significant difference in the $E$ with the optimal control $u_{1}^{*}$ and $u_{2}^{*}$ compared to exposed subpopulation without control, using effective controls decreased the number of exposed COVID-19 (E) than without control. 


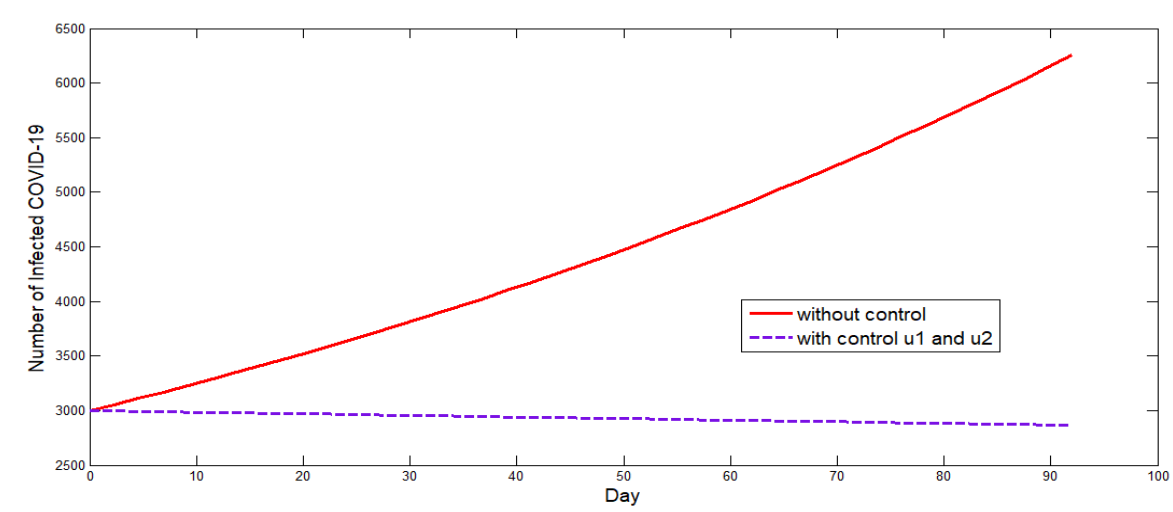

Figure 2. The dynamics of $I$ with $u_{1}^{*}$ and $u_{2}^{*}$ controls

Figure 2, the optimal control $\left(u_{1}^{*}\right)$ and $\left(u_{2}^{*}\right)$ on COVID-19 are used to equation (12). The results in Figure 2 show that there is difference in the $I$ with control than $I$ without control $u_{1}^{*}$ and $u_{2}^{*}$, using effective controls decreased the number of active COVID-19 (I) compared to without control.

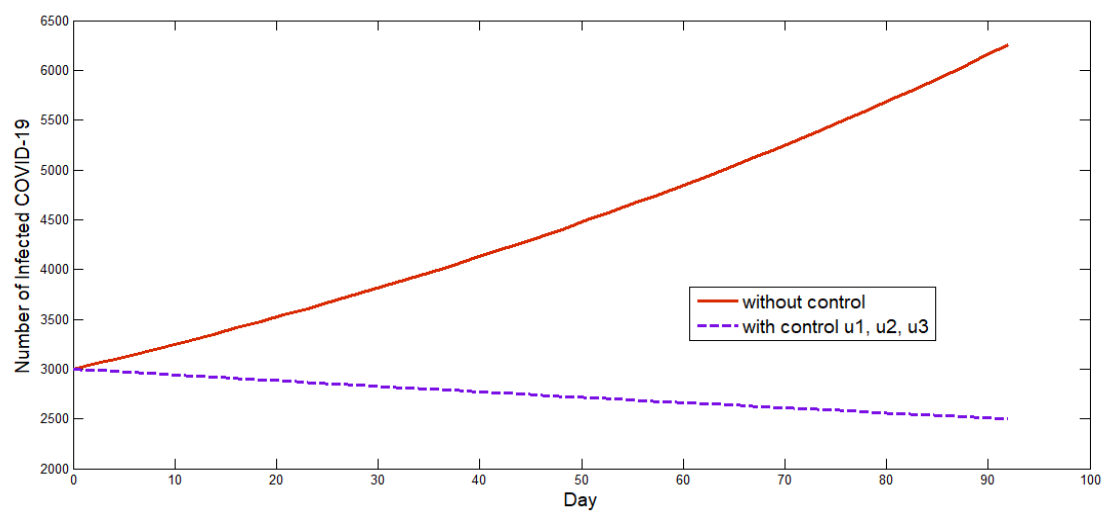

Figure 3. The dynamics of $I$ with $u_{1}^{*}$ and $u_{2}^{*}$ controls

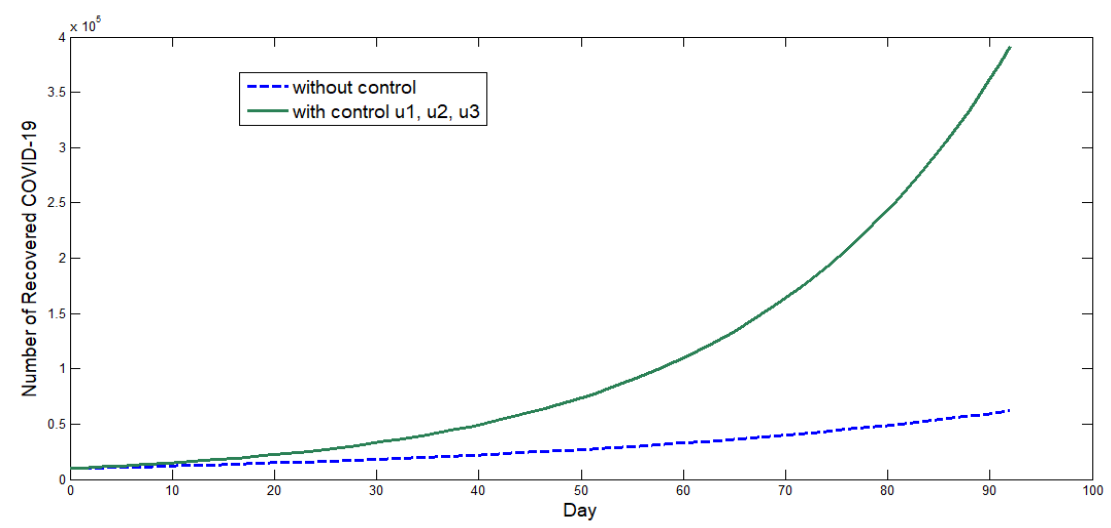

Figure 4. The dynamics of $R$ with $u_{1}^{*}, u_{2}^{*}$ and $u_{3}^{*}$ control

Figure 3, the optimal control on COVID-19 prevention $\left(u_{1}^{*}\right)$, the optimal control $\left(u_{2}^{*}\right)$ and optimal control on COVID-19 treatment $\left(u_{3}^{*}\right)$ are application equation (12). The results in Figure 3 show that there is difference in the $I$ with control than $I$ without control $u_{1}^{*}, u_{2}^{*}$ and $u_{3}^{*}$ compared to $I$ without control, using effective controls decreased the number of active Covid-19 (I) compared to with control strategy $u_{1}^{*}$ and $u_{2}^{*}$ and without control. 
Figure 4, the results in Figure 4 show that a significant difference in the $R$ with the optimal control strategy $u_{1}^{*}, u_{2}^{*}$ and $u_{3}^{*}$ compared to $R$ without control. We see in Figure 4 The number of recovered individuals in subpopulation of COVID-19 increases rapidly with optimal control, while it is increase slowly without the control.

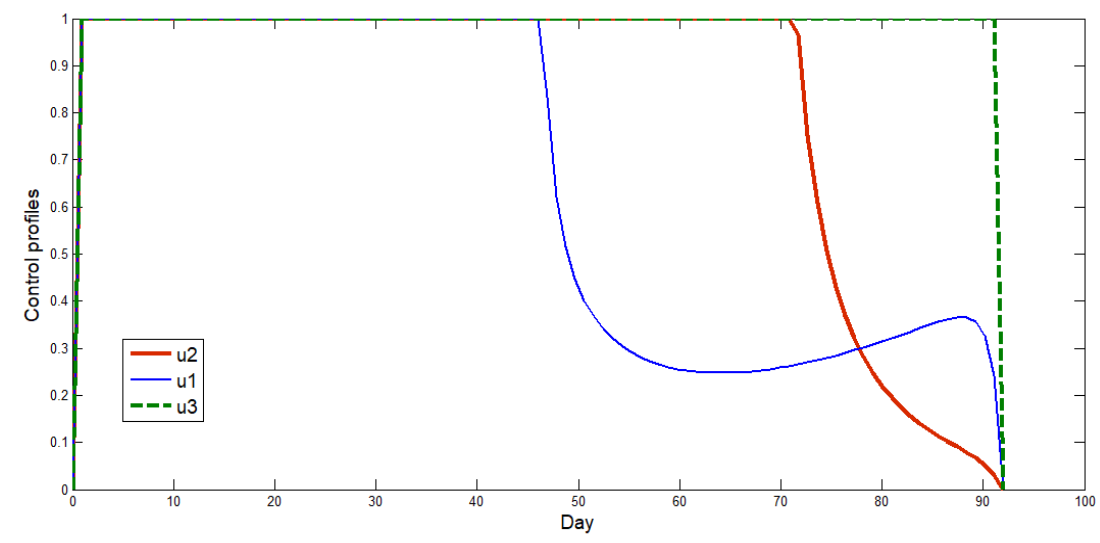

Figure 5. The profile of the optimal controls $u_{1}^{*}, u_{2}^{*}$ and $u_{3}^{*}$

Figure 5: in this scenario, we consider the COVID-19 optimal prevention and treatment control of COVID-19 simultaneously. The profile of the optimal prevention control $u_{1}^{*}, u_{2}^{*}$ and optimal treatment control $u_{3}^{*}$ of this scenario in figure 5 .

\section{CONCLUSION}

Until now, various efforts have been made by medical personnel in each country and WHO has been trying to find a cure and a vaccine for Covid-19, but until now it has not been found so that the spread of covid-19 in the world has not been controlled.

But in early 2021 a vaccine for COVID-19 has been found and vaccinations have been carried out all over the world. Having been vaccinated against COVID-19 does not guarantee that you will not be infected with COVID-19 again. The model studied in this paper discusses model COVID-19 in Indonesia concerning vaccinations. Based on the numerical simulation obtained optimal control strategy $u_{1}^{*}$ and $u_{2}^{*}$ compared to $E$ without control, using effective controls decreased the number of exposed Covid-19 $(E)$ compared to without control. optimal control strategy $u_{1}^{*}, u_{2}^{*}$ and $u_{3}^{*}$ compared to $I$ without control, using effective controls decreased the number of active Covid-19 $(I)$ compared to with control strategy $u_{1}^{*}$ and $u_{2}^{*}$ and without control. The control result in a further increase in the number who recovered of covid-19 $(R)$ compared optimal control strategy $u_{1}^{*}$ and $u_{2}^{*}$ and without control.

\section{ACKNOWLEDGMENTS}

The authors would like to thank Kemenristek Dikti for providing Higher Education Grants for Fiscal Year 2020, through LPPM Universitas Cenderawasih who sponsored the research. 


\section{REFERENCES}

[1] Kemenkes RI, "Dokumen resmi," Pedoman kesiapan menghadapi COVID-19, pp. 0$115,2020$.

[2] E. Jung, S. Iwami, Y. Takeuchi, and T. C. Jo, "Optimal control strategy for prevention of avian influenza pandemic," J. Theor. Biol., vol. 260, no. 2, pp. 220-229, 2009.

[3] M. Veera Krishna, "Mathematical modeling on diffusion and control of COVID-19," Infect. Dis. Model., vol. 5, pp. 588-597, 2020.

[4] S. S. Moses, S. Qureshi, S. Zhao, A. Yusuf, U. T. Mustapha, and D. He, "Mathematical modeling of COVID-19 epidemic with effect of awareness programs," Infect. Dis. Model., vol. 6, no. February, pp. 448-460, 2021.

[5] J. Arino and S. Portet, “A simple model for COVID-19," Infect. Dis. Model., vol. 5, pp. 309-315, 2020.

[6] M. Kamrujjaman, M. S. Mahmud, and M. S. Islam, "Coronavirus Outbreak and the Mathematical Growth Map of COVID-19," Annu. Res. Rev. Biol., no. March, pp. 72-78, 2020.

[7] M. Imran, M. Wu, Y. Zhao, E. Beşe, and M. J. Khan, "Mathematical Modelling of SIR for COVID-19 Forecasting,” vol. XXX, no. February, pp. 218-226, 2021.

[8] S. K. Biswas, J. K. Ghosh, S. Sarkar, and U. Ghosh, "COVID-19 pandemic in India: a mathematical model study," Nonlinear Dyn., vol. 102, no. 1, pp. 537-553, 2020.

[9] J. Tian et al., "Modeling analysis of COVID-19 based on morbidity data in Anhui, China," Math. Biosci. Eng., vol. 17, no. 4, pp. 2842-2852, 2020.

[10] Y. Fang, Y. Nie, and M. Penny, "Transmission dynamics of the COVID-19 outbreak and effectiveness of government interventions: A data-driven analysis," J. Med. Virol., vol. 92, no. 6, pp. 645-659, 2020.

[11] Y. Li et al., "Mathematical Modeling and Epidemic Prediction of COVID-19 and Its Significance to Epidemic Prevention and," Ann. Infect. Dis. Epidemiol., vol. 5, no. 1, p. 1052, 2020.

[12] C. Y. Shen, "Logistic growth modelling of COVID-19 proliferation in China and its international implications," Int. J. Infect. Dis., vol. 96, pp. 582-589, 2020.

[13] M. Tomochi and M. Kono, "A mathematical model for COVID-19 pandemic-SIIR model: Effects of asymptomatic individuals," J. Gen. Fam. Med., vol. 22, no. 1, pp. 5$14,2021$.

[14] R. ud Din, A. R. Seadawy, K. Shah, A. Ullah, and D. Baleanu, "Study of global dynamics of COVID-19 via a new mathematical model," Results Phys., vol. 19, p. 103468, 2020.

[15] J. Ma, "Estimating epidemic exponential growth rate and basic reproduction number," Infect. Dis. Model., vol. 5, pp. 129-141, 2020.

[16] R. Cherniha and V. Davydovych, "A mathematical model for the COVID-19 outbreak and its applications," Symmetry, Basel, vol. 12, no. 6, 2020.

[17] S. Lenhart and J. T. Workman, Optimal Control Applied to Biological Models, John Chapman and Hall, New York, 2007. 